Journal of Advanced Research in Fluid Mechanics and Thermal Sciences

\title{
Simulation of Water Wave Interaction with Large Submerged Square Obstacles
}

\author{
Mona Gomaa ${ }^{1, *}$, Tamer Kasem ${ }^{1,2, *}$ \\ Smart Engineering Systems Research Center (SESC), Nile University, Egypt \\ Cairo University, Egypt
}

\begin{tabular}{ll} 
ARTICLE INFO & ABSTRACT \\
\hline $\begin{array}{l}\text { Article history: } \\
\text { Received } 10 \text { May } 2021\end{array}$ & $\begin{array}{l}\text { Water waves propagation over submerged obstacles is considered. The problem serves } \\
\text { as an efficient model for modeling breakwaters. A numerical wave tank is developed } \\
\text { to simulate the induced flow field. The model is based on multiphase viscous flow } \\
\text { Accepted } 10 \text { July } 2021\end{array}$ \\
$\begin{array}{l}\text { Available online } 12 \text { August } 2021 \\
\text { assumptions. Computations are performed adopting clustered grids and suitable initial } \\
\text { and boundary conditions. The results are verified using the flow field particle image } \\
\text { velocimetry (PIV) measurements. Spatial and temporal resolutions are validated. } \\
\text { Complex flow phenomena occurring due to the presence of the relatively large sized } \\
\text { obstacle are visualized. The effect of wave parameters on the flow structure is } \\
\text { Multiphase; breakwater; wave forces; }\end{array}$ & $\begin{array}{l}\text { investigated. A brief parametric study is presented and the resultant wave forces and } \\
\text { turning moments are provided. }\end{array}$
\end{tabular}

\section{Introduction}

Breakwaters are common maritime structures constructed for shore protection [1]. A special type of these structures is submerged breakwaters. It is generally characterized by being low crested (height beneath sea free surface). Submerged breakwaters enjoy an aesthetic advantage since the clear sea view is not interrupted [2]. Breakwaters must endure hostile sea conditions, and their accurate simulation is crucial. Among various environmental phenomena, water waves possess the greatest influence, and hold the highest importance [1]. In addition water waves have a strong potential for energy harvesting [3].

The problem of wave propagation over obstacles serves as an efficient model for breakwater applications [4]. Two specific challenges of the problem are reported [5]. The first challenge is the strong vortex shedding occurring near the obstacle. Vortices have strong influence on the flow field, due to generated turbulence. Accurate modelling of this rotational flow field is necessary due to its

\footnotetext{
* Corresponding author.

E-mail address: MoGomaa@nu.edu.eg

* Corresponding author.

E-mail address: tkasem@nu.edu.eg
}

https://doi.org/10.37934/arfmts.86.1.1426 
impact on sediment motion near a submerged breakwater. The second challenge is the strong free surface deformation occurring for relatively large sized obstacles [6].

Considerable literature is available focusing on the problem considered. The interested reader may refer to [5], where it was concluded that the problem is best modelled adopting viscous and multiphase assumptions. Development of such models is a nontrivial task. Numerous discretization points are needed to achieve adequate resolution. Hence special iterative solvers are needed to solve the huge algebraic systems. In addition, the domain size should be long enough to avoid boundary reflected waves. Boundary conditions should be carefully imposed at inlet to obtain suitable incident wave. Unless suitable grid clustering is adopted, transient simulations will consume prohibitively long times. For instance, grid clustering can be employed near high gradients regions to reduce computational requirements.

Faced by these challenges a numerical wave tank (NWT) based on ANSYS commercial package [7, 8] is adopted in the current work. This package is rather reliable and was adopted in many modern studies [9]. Details of model development is well described. Grid clustering near the free surface and the square obstacle is performed to avoid artificial numerical dissipation. The unstructured grid employed is crucial for the current application and other complex ones [10]. The crucial aspect of imposing balanced turbulence initial conditions is well emphasized.

An important aspect of the current work is model validation. This is often achieved using water free surface level and pressure measurements $[5,6,11]$. However, comparisons with velocity field measurements needed to verify the vortex dynamics are missing even in recent publications [12-14]. A few years ago, flow field velocity measurements were provided using the Particle Image Velocimetry (PIV) method [15]. The experiments were done adopting a square obstacle influenced by periodic water waves. The measurements covered a continuous two-dimensional window. Hence the vortical flow field should have been well captured. This data set presents a corner stone in the current work. The results of the developed numerical wave tank will be verified using horizontal and vertical velocity profiles. Spatial and temporal resolutions will be elucidated.

Once model validation is achieved, the complete flow field is visualized, and the flow features are clarified. The vortex generation, evolution and dissipation process are presented. A brief parametric study is provided where the influence of wave parameters is clarified. Finally, design features including wave forces and moments are presented.

\section{Governing Equations}

The model assumptions are incompressible, multiphase, and turbulent flow. The governing equations are provided below.

$\frac{\partial u}{\partial x}+\frac{\partial u}{\partial y}=0$

$\rho\left(\frac{\partial u}{\partial t}+u \frac{\partial u}{\partial x}+v \frac{\partial u}{\partial y}\right)=-\frac{\partial p}{\partial x}+\left(\mu_{t}+\mu\right)\left(\frac{\partial^{2} u}{\partial x^{2}}+\frac{\partial^{2} u}{\partial y^{2}}\right)$

$\rho\left(\frac{\partial v}{\partial t}+u \frac{\partial v}{\partial x}+v \frac{\partial v}{\partial y}\right)=-\frac{\partial p}{\partial y}-\rho g+\left(\mu_{t}+\mu\right)\left(\frac{\partial^{2} v}{\partial x^{2}}+\frac{\partial^{2} v}{\partial y^{2}}\right)$

$\frac{\partial}{\partial t}(\rho k)+\frac{\partial}{\partial x_{i}}\left(\rho k u_{i}\right)=\frac{\partial}{\partial x_{j}}\left(\Gamma_{k} \frac{\partial k}{\partial x_{j}}\right)+G_{k}-Y_{k}+S_{k}$ 


$$
\begin{aligned}
& \frac{\partial}{\partial t}(\rho \omega)+\frac{\partial}{\partial x_{i}}\left(\rho \omega u_{i}\right)=\frac{\partial}{\partial x_{j}}\left(\Gamma_{\omega} \frac{\partial \omega}{\partial x_{j}}\right)+G_{\omega}-Y_{\omega}+S_{\omega} \\
& \frac{\partial}{\partial t}(\alpha \rho)+\nabla \cdot(\alpha \rho \bar{v})=0 \\
& \rho=\alpha \rho_{2}+(1-\alpha) \rho_{1}
\end{aligned}
$$

Here Eq. (1) is the continuity equation, Eq. (2) and (3) are the momentum equations for two dimensional flow. Eq. (4) and (5) represent the turbulence model. Eq. (6) is the volume fraction equation used to track the air-water interface. The volume fraction $\alpha$ has three possible values. The limiting values $\alpha=0$ or $\alpha=1$ indicate a cell that is totally occupied by air or water, respectively. A value in the range of $0<\alpha<1$ indicates an interface cell. In two-phase flow the fluid properties used in the transport equations $(\rho$ and $\mu$ ) are determined in the same manner as density in Eq. (7).

Values of fluid properties were selected corresponding to water properties: $\rho_{2}=998.2 \mathrm{~kg} \mathrm{~m}^{-3}$ and $\mu_{2}=1.003 \times 10^{-3} \mathrm{~kg} \mathrm{~m}^{-1} \mathrm{~s}^{-1}$, air properties: $\rho_{1}=1.225 \mathrm{~kg} \mathrm{~m}^{-3}$ and $\mu_{1}=1.7894 \times$ $10^{-5} \mathrm{Kg} \mathrm{m}^{-1} \mathrm{~s}^{-1}$.

The SST $k-\omega$ turbulence model is adopted since it generally yields accurate predictions of pressure-induced separation vortices [16]. In the above equations $G_{k}$ and $G_{\omega}$ represent the generation of turbulence kinetic energy $k$ and $\omega$, respectively. The effective diffusivity of $k$ and $\omega$ is modeled by $\Gamma_{k}$ and $\Gamma_{\omega}$, respectively. Turbulence dissipation of $k$ and $\omega$ is modeled by $Y_{k}$ and $Y_{\omega}$, respectively. Finally, $\mu_{t}$ is defined as $\mu_{t}=\rho \frac{k}{\omega}$.

\subsection{Boundary Conditions}

Nonlinear Stokes second-order wave enter the domain from the left side. Generally, slip velocity conditions are imposed on walls, except on the submerged obstacle. A numerical beach is implemented on the last fifth part of the NWT. This is necessary to avoid boundary-reflected waves. Specifically, linear, and quadratic expressions in terms of the velocity are added to the momentum equation. They serve as a damping mechanism. The coefficients of the linear and the quadratic terms are assigned to $100 \mathrm{~s}^{-1}$ and $100 \mathrm{~m}^{-1}$, respectively. The enhanced wall treatment developed in [17] is adopted to impose turbulence boundary conditions. This setup avoids the requirement of extremely small sized grid near walls.

\subsection{Initial Conditions}

To avoid excessive wave dissipation, the following aspects need to be considered [18]

i. The initial values of $k$ and $\omega$ should be high enough to avoid instability.

ii. The resulting kinematic eddy viscosity $v_{t}=\frac{k}{\omega}$ should be low enough to avoid extra damping.

Based on numerical experiments the following initial values are adopted $k=1 \times 10^{-3} \mathrm{~m}^{2} \mathrm{~s}^{-2}$ and $\omega=500 s^{-1}$. The corresponding initial $\mu_{t}$ is of the same order of $\mu_{2}$. Accurate results based on the values above will be presented shortly. 


\section{General Mesh Setup}

The rectangular computational domain is discretized with a structured grid away from obstacles (Figure 1). A clustered layer has been generated at the air-water interface to catch sudden velocity changes. This is implemented by the option of mesh bias growth rate (BGR) available in ANSYS.

As already mentioned, slip conditions are applied at walls. Hence, relatively coarse mesh is adopted near them. Finally, a numerical beach zone occupies the last fifth portion of the domain adjacent to the exit. Relatively coarse meshing can be employed in the numerical beach zone. Mesh clustering should be employed carefully to avoid neither too large, nor too small cell aspect ratios.

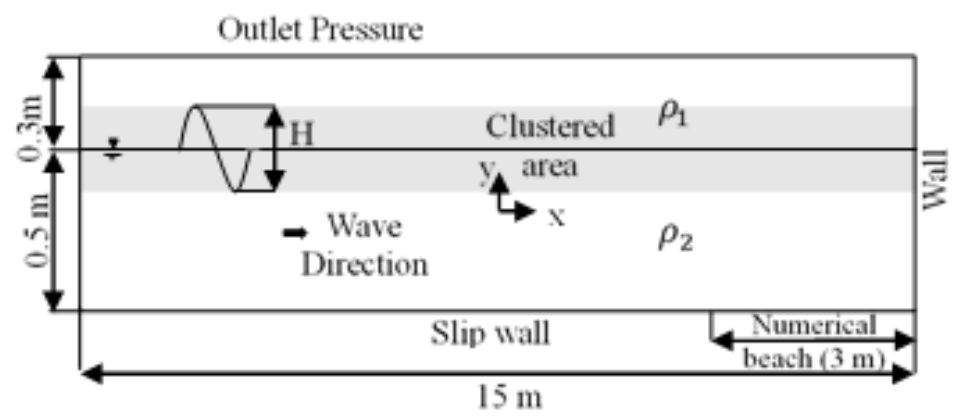

Fig. 1. NWT computational domain (not to scale) used to simulate flat bottom propagation

\section{Numerical Scheme}

The solution scheme options used in all cases are listed in Table 1.

Table 1

Solution methods

\begin{tabular}{ll}
\hline Solution methods & Setting \\
\hline Pressure interpolation method & PRESTO \\
Interpolation gradient method & Least squares cell-based \\
Momentum discretization methods & Second order upwind \\
Transient formulation & Second order implicit \\
\hline
\end{tabular}

\section{Results}

\subsection{Flat Bottom NWT Validation}

The numerical wave tank (NWT) is validated via simulation of wave propagation over a flat bottom. Simulation of this relatively simple test case is useful since analytical solutions are available for validation. Stokes second order theoretical solution is given as follows. The velocity field is given as [19]

$$
\begin{aligned}
& u=\frac{H}{2} \frac{g \kappa}{\sigma} \frac{\cosh \kappa(h+y)}{\cosh \kappa h} \cos (\kappa x-\sigma t)+\frac{3}{16} \frac{H^{2} \sigma \kappa \cosh 2 \kappa(h+y)}{\sinh ^{4} \kappa h} \cos 2(\kappa x-\sigma t) \\
& v=\frac{H}{2} \frac{g \kappa}{\sigma} \frac{\sinh \kappa(h+y)}{\cosh \kappa h} \sin (\kappa x-\sigma t)+\frac{3}{16} \frac{H^{2} \sigma \kappa \sinh 2 \kappa(h+y)}{\sinh ^{4} \kappa h} \sin 2(\kappa x-\sigma t)
\end{aligned}
$$


In the above equations $H$ is the wave height (from crest to trough), $\sigma=2 \Pi / T$ is the frequency defined in terms of the wave period $T, \kappa=2 \Pi / L$ is the wave number defined in terms of the wave length $L, h$ is the water depth, and $y$ is the vertical coordinate positive upward and measured from the still water level. Stokes second order solution is limited to the liquid phase, excluding the gas phase. Hence the equation is valid up to the free surface.

Various relevant options available in ANSYS were examined. Specifically, the effects of mesh bias growth rate (BGR), total number of elements, and free surface turbulence damping were tested. A comparison between three different cases was done to decide which BGR will be adopted for the rest of the analysis.

Several setups are adopted to determine the effect of BGR, the different cases conditions are listed in Table 2. For the three cases, the incoming wave parameters are selected as: $H=$ $0.05 m, T=1.569 \mathrm{~s}, L=3 \mathrm{~m}$ and $h=0.5 \mathrm{~m}$. As obvious in Figure 2 there is no difference between the three cases. Hence parameters of cases 2 and 3 should be favored to minimize the solution time.

Table 2

Conditions of different cases

\begin{tabular}{llll}
\hline Conditions & Case1 & Case 2 & Case 3 \\
\hline Bias at the vertical direction & 1.2 & 2 & 2 \\
No. Elements & 35000 & 24000 & 24000 \\
Turbulence damping option & No & No & Yes \\
\hline
\end{tabular}

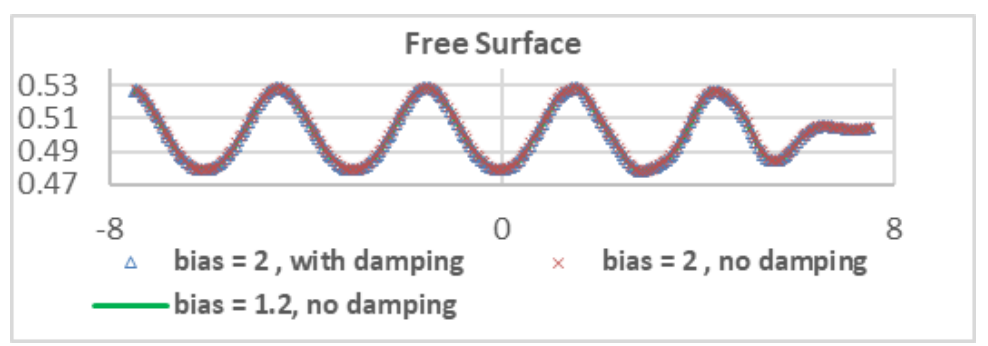

Fig. 2. Free surface level of the three cases after 18 seconds

Figure 3 and 4 represent the velocity profiles ( $u$ and $v$ ) at equally spaced phases in one period. Strong agreement between theoretical and numerical results is clarified. The spatial resolution in the vertical direction and temporal resolution during a typical wave period are illustrated.

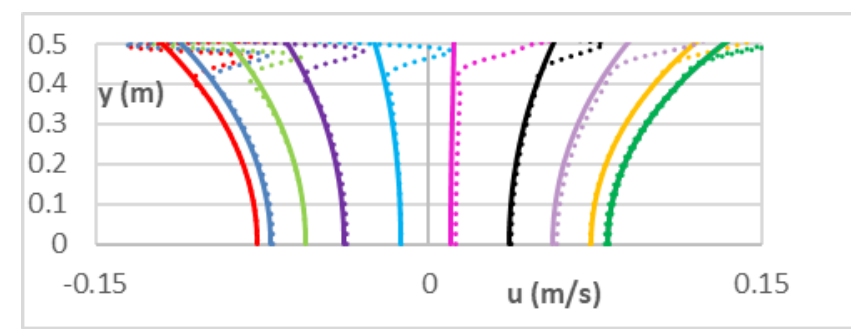

Fig. 3. Velocity profiles (u vs y) for numerical solution (dotted lines) and analytical solution (solid lines) 


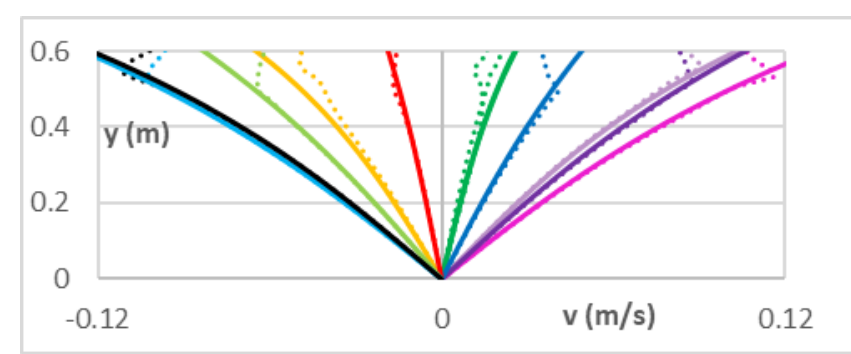

Fig. 4. velocity profiles ( $v$ vs y) for Numerical solution (dotted lines) and analytical solution (solid lines)

\subsection{Square Obstacle}

In Figure 5 the dimensions of the square obstacle case domain are represented, with a measurement window of height $0.12 \mathrm{~m} \times$ width $0.1 \mathrm{~m}$. The window is located directly behind the obstacle as shown. Considerable grid clustering is employed in the vicinity of the obstacle

Experimental measurements were obtained using the particle image velocimetry technique (PIV). Full details are provided in [15]. However, a brief description is given below to provide a concise presentation. The experiments are conducted using the $24 \mathrm{~m}$ length wave flume located at Bergische Universität Wuppertal. The water depth $h$ was fixed at $0.3 \mathrm{~m}$ for all runs. The measurement window was populated with neutrally buoyant particles, whose density is close to that of water. Hence these particles should move with almost the same water velocity. Using suitable lighting, videos are recorded at high speed of 120 frames per second. The videos are analyzed using the free software MatPIV [20].

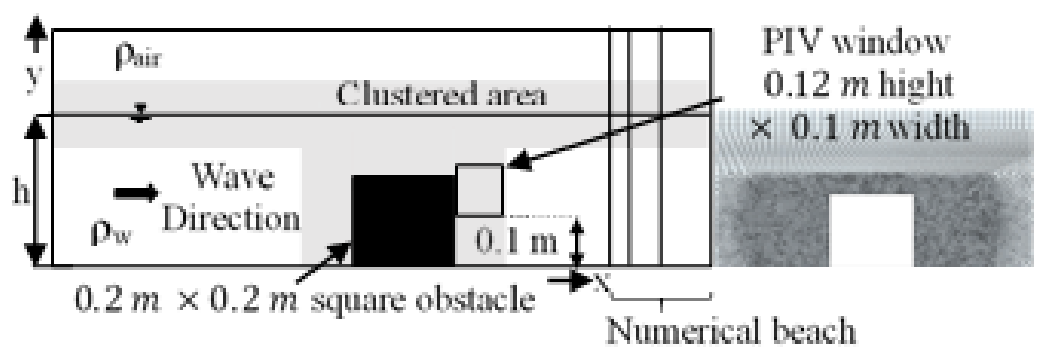

Fig. 5. Square obstacle domain

The various wave parameters for all cases are shown in the Table 3. The CFD results for cases 3 , 8 and 9 are compared with the wave induced flow behind submerged obstacles by [15].

Table 3

Wave Parameters for different nine cases

\begin{tabular}{llll}
\hline Case No. & $\mathrm{H}(\mathrm{m})$ & $\mathrm{T}(\mathrm{sec})$ & $\mathrm{L}(\mathrm{m})$ \\
\hline 1 & 0.02 & 0.8 & 0.96 \\
2 & 0.03 & 0.8 & 0.96 \\
3 & 0.04 & 0.8 & 0.96 \\
4 & 0.02 & 0.9 & 1.16 \\
5 & 0.03 & 0.9 & 1.16 \\
6 & 0.04 & 0.9 & 1.16 \\
7 & 0.02 & 1.0 & 1.37 \\
8 & 0.03 & 1.0 & 1.37 \\
\hline
\end{tabular}


The horizontal velocity profiles are drawn at $x=0.01 \mathrm{~m}$ vertical line, and the vertical velocity profiles are drawn at $y=0.08 \mathrm{~m}$ horizontal line. The origin is selected at the bottom left corner of the measurement window. The window position is selected to detect a downstream vortex. The vortex starts to develop as the wave crest approaches the obstacle. The crest induces high horizontal velocity in the positive direction above the obstacle. This stage can be observed at phase $120^{\circ}$ in Figure 6,7 and 8 . The sharp velocity gradient results in the generation of a clockwise vortex. The positive and negative velocities of the vorticial flow field ate well detected in phase $180^{\circ}$ of Figure 6 , 7 and 8. Finally, as the wave trough arrives the vortex diminishes. This is due to the reversed flow direction (to the negative) at the obstacle top. This stage is well observed in phase $360^{\circ}$ of Figure 6 , 7 and 8 . Similar observations can be drawn for the vertical velocity profiles presented in Figure 9, 10 and 11 .

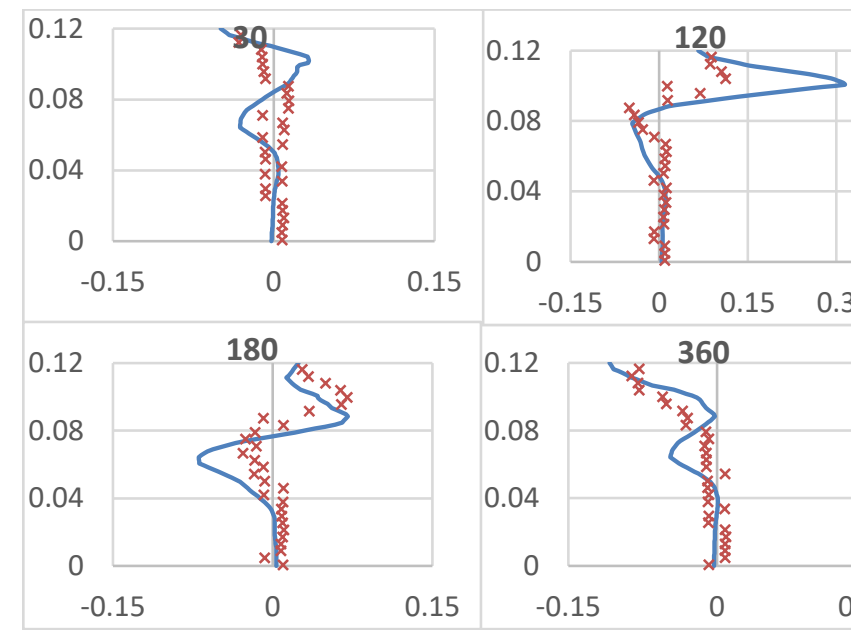

Fig. 6. Velocity profiles (u vs y) behind a square obstacle, Case 3- Experimental (crosses) and Numerical (solid line)

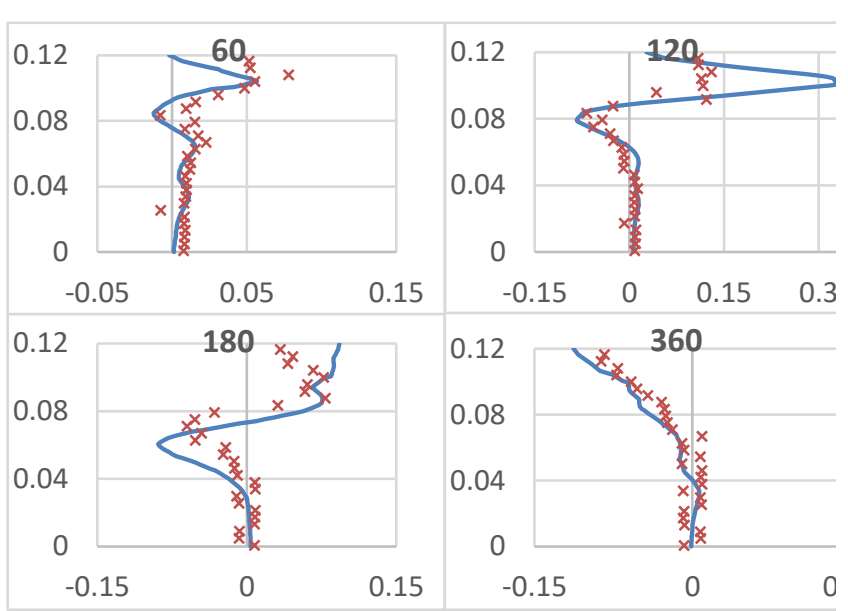

Fig. 7. Velocity profiles (u vs y) behind a square obstacle, Case 8- Experimental (crosses) and Numerical (solid line)

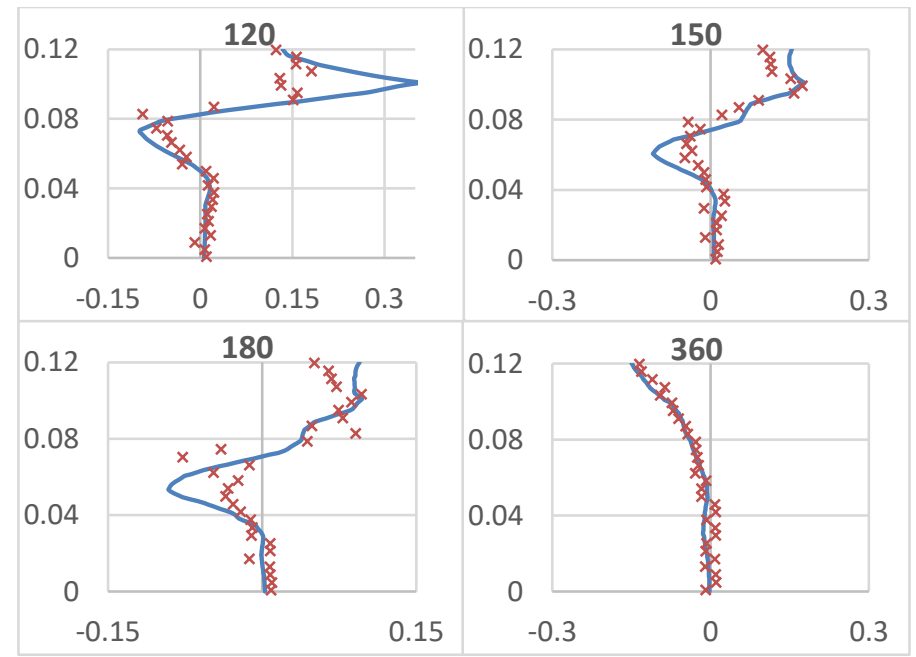

Fig. 8. Velocity profiles (u vs y) behind a square obstacle, Case 9- Experimental (crosses) and Numerical (solid line)

The well agreement between PIV measurements and NWT calculations is rather clear. However, some discrepancy is present at sudden accelerated instants. This can be observed for instance at phase $120^{\circ}$ in Figure 6, 7 and 8. This discrepancy may be attributed to the phase lag of PIV data. Generally, PIV particles cannot exactly follow the fluid motion, specially at high fluid accelerations. 
The proportional relation between maximum vortex velocities and both wave period $T$ and height $H$ can be observed. This is expected since the wave energy is proportional to both parameters.

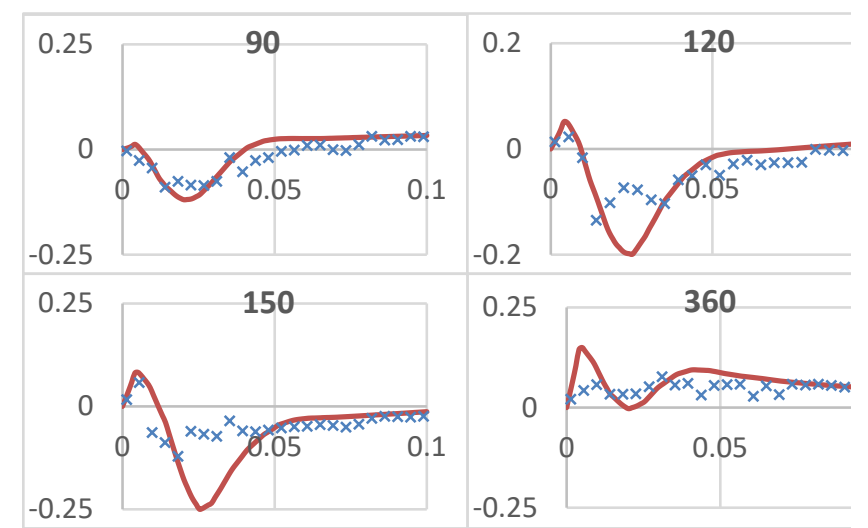

Fig. 9. Velocity profiles ( $v$ vs $x$ ) behind a square obstacle, Case 3- Experimental (crosses) and Numerical (solid line)

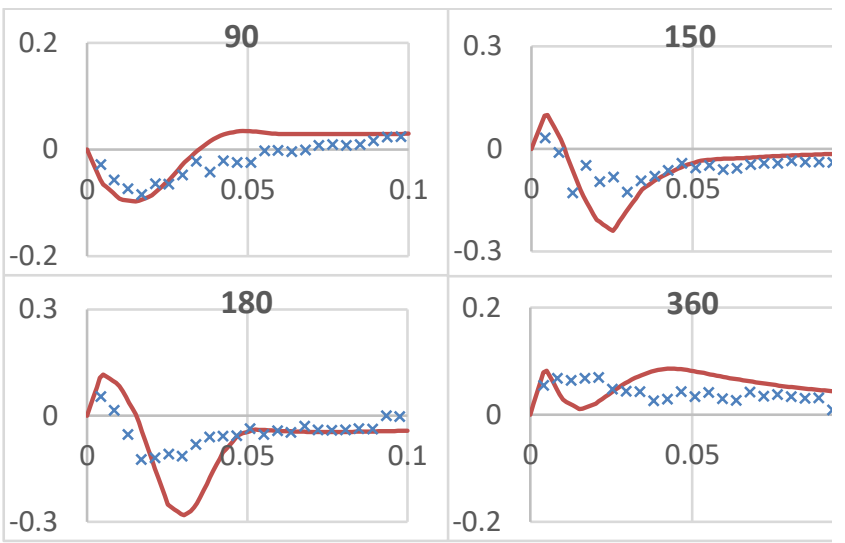

Fig. 10. Velocity profiles ( $v$ vs $x$ ) behind a square obstacle, Case 8- Experimental (crosses) and Numerical (solid line)

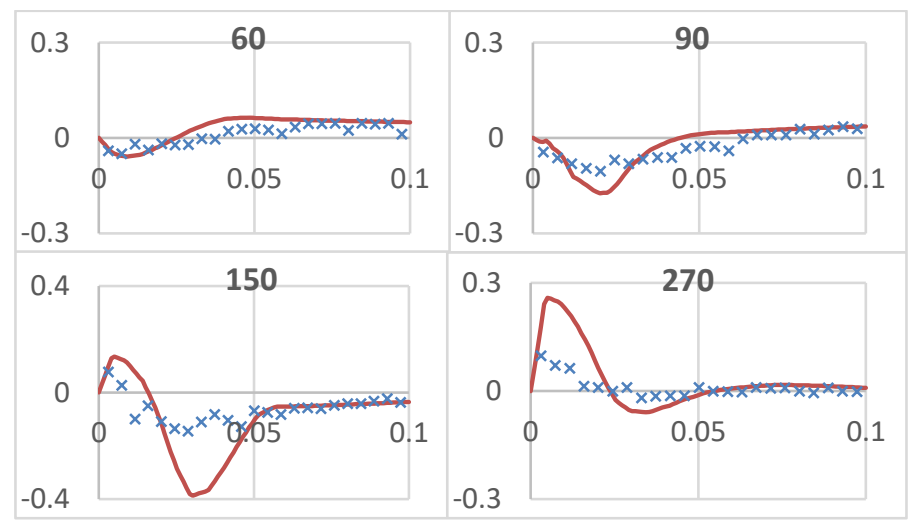

Fig. 11. Velocity profiles ( $v$ vs $x$ ) behind a square obstacle, Case 9- Experimental (crosses) and Numerical (solid line)

Figure 12-14 shows streamlines and free surface profiles at six significant phases for cases 3,8 and 9, respectively. Zoomed figures for vortices located at various locations are displayed. A common feature for the three cases is the free surface steepening as the wave crest crosses the obstacle. This strong free surface deformation is expected for the large sized obstacle discussed.

Four distinct vortices are observed. At the top left corner, one vortex evolves adjacent to the horizontal upper surface $\left(V_{L H}\right)$, and another one adjacent to upstream vertical surface $\left(V_{L V}\right)$. Similarly, $V_{R H}$ and $V_{R V}$ are defined at the right corner. The sizes of $V_{L H}$ and $V_{R H}$ are smaller compared to $V_{L V}$ and $V_{R V}$, respectively. At each corner vortices evolve in alternating behavior. This is clarified for instance in 13 , as once $V_{L H}$ vanishes at phase $60^{\circ}, V_{L V}$ is observed at phase $150^{\circ}$. At each corner, the vortices rotate in opposite directions. Specifically, $V_{L H}$ and $V_{L V}$ rotate in clockwise and anti-clock directions, respectively. The situation is mirrored at the right corner, where $\mathrm{V}_{\mathrm{RV}}$ and $\mathrm{V}_{\mathrm{RH}}$ rotate in clockwise and anti-clock directions, respectively. It is expected that most of the wave energy is dissipated at the downstream side, where strong mixing occurs after the wave crosses the obstacle. This hypothesis is supported by simulation results, since $V_{R V}$ is characterized by the biggest size among the total four vortices. 


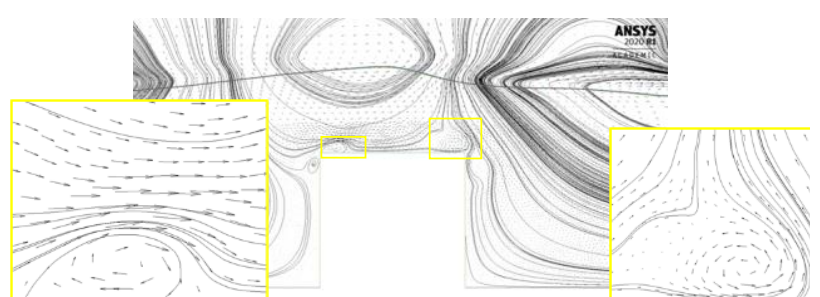

$30^{\circ}$

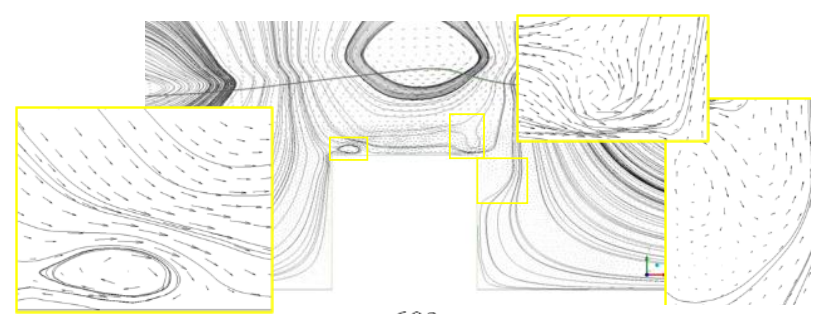

$60^{\circ}$

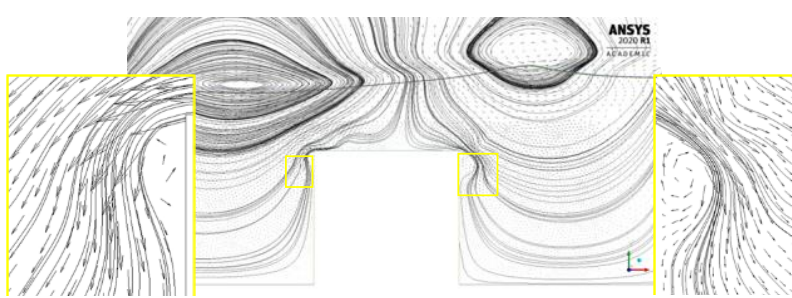

$150^{\circ}$

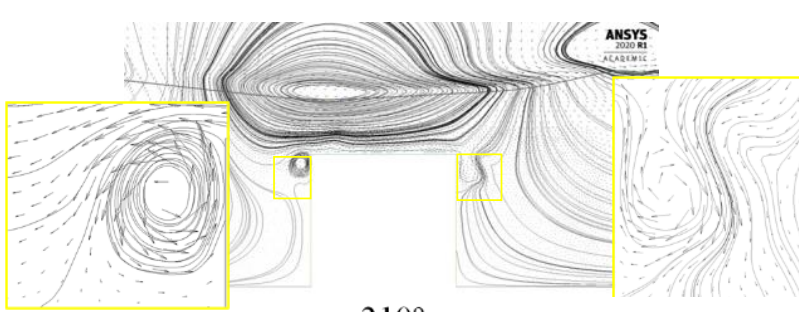

$210^{\circ}$

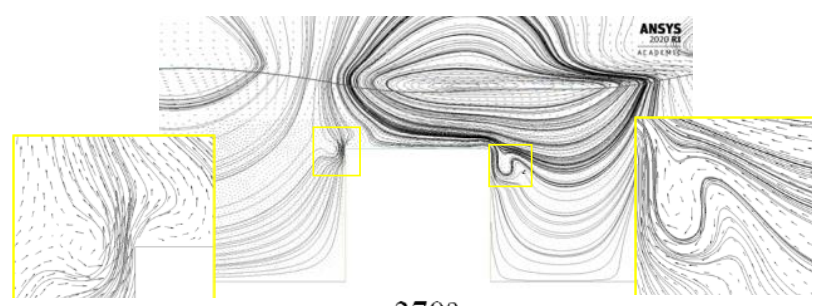

$270^{\circ}$

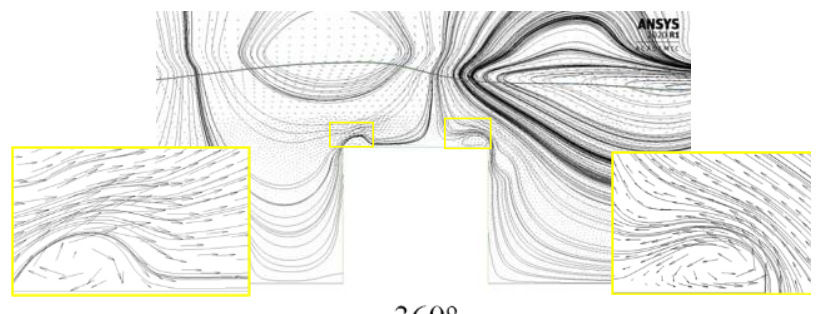

$360^{\circ}$

Fig. 12. Streamlines at 6 significant phases, case 3

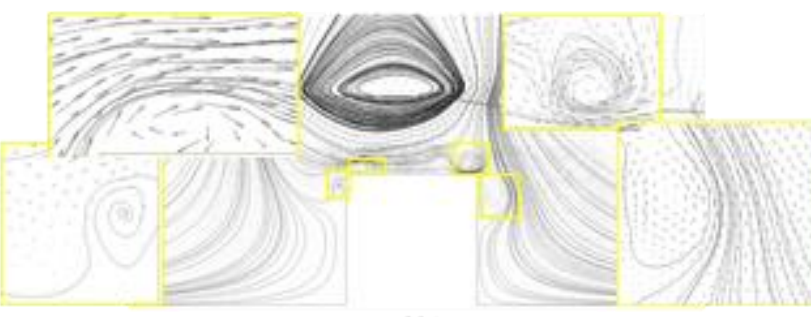

$60^{\circ}$

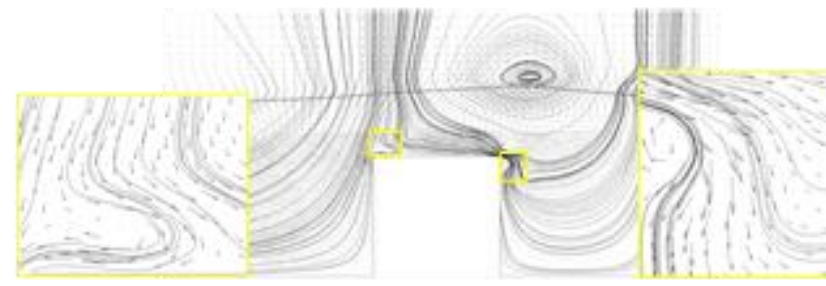

$120^{\circ}$
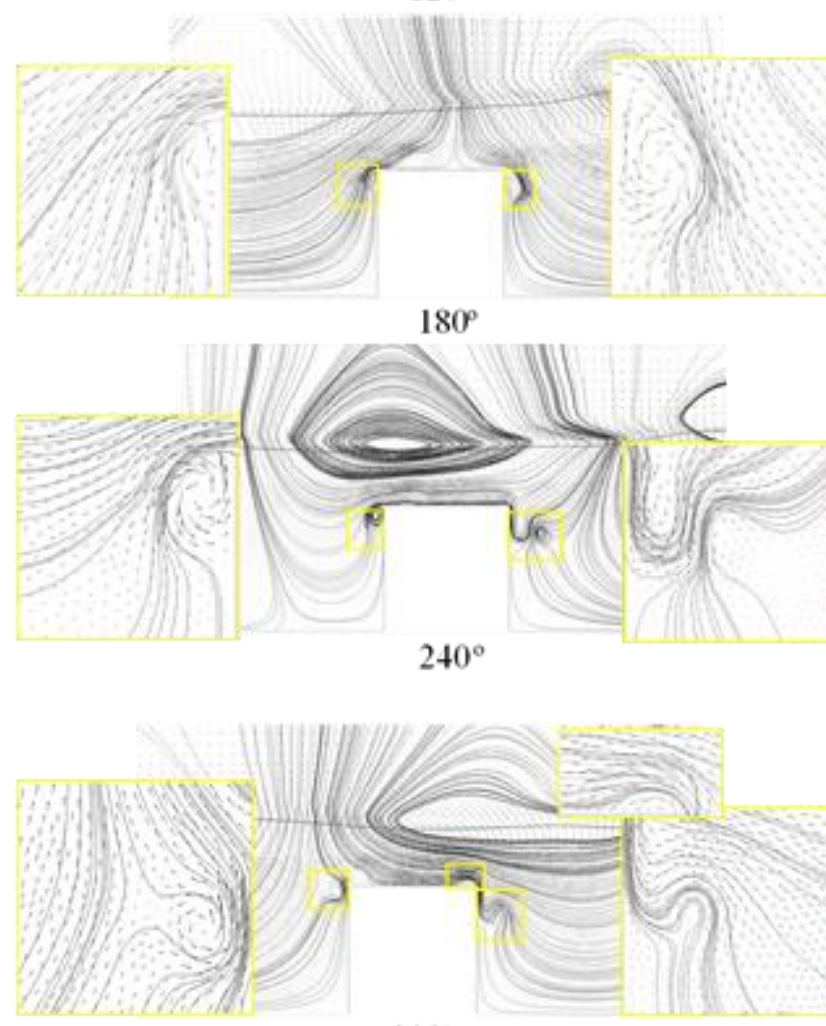

$300^{\circ}$

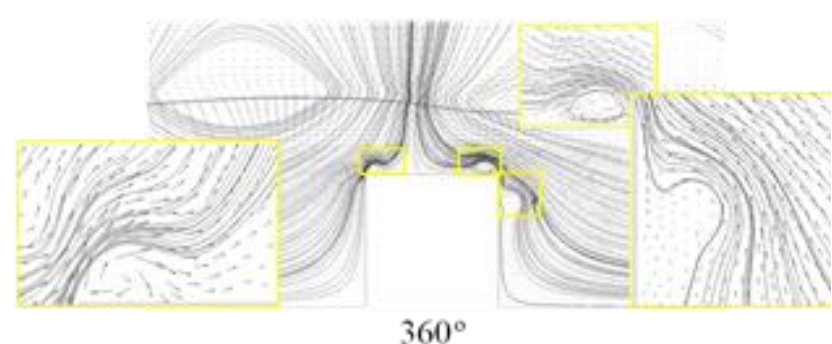

Fig. 13. Streamlines at 6 significant phases, case 8 


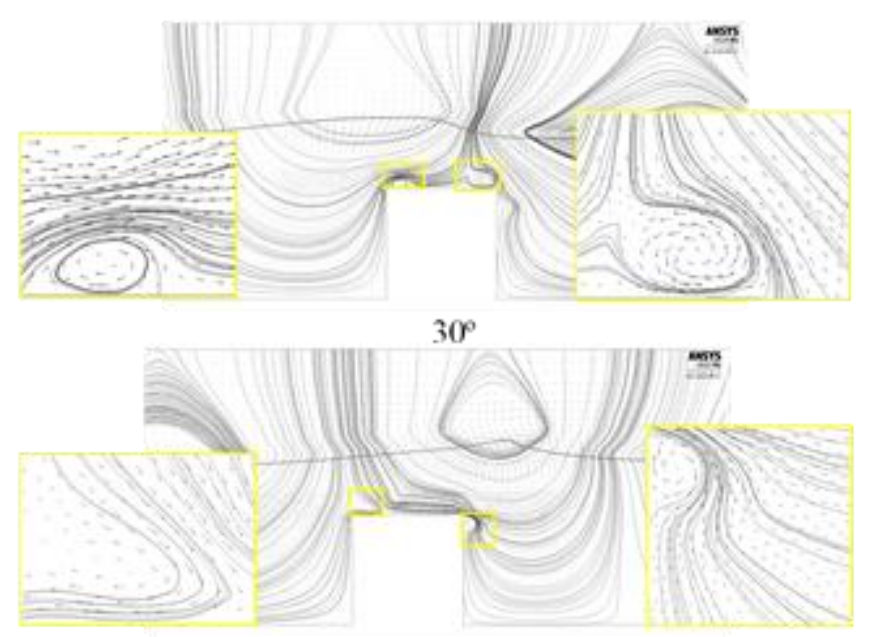

$120^{\circ}$

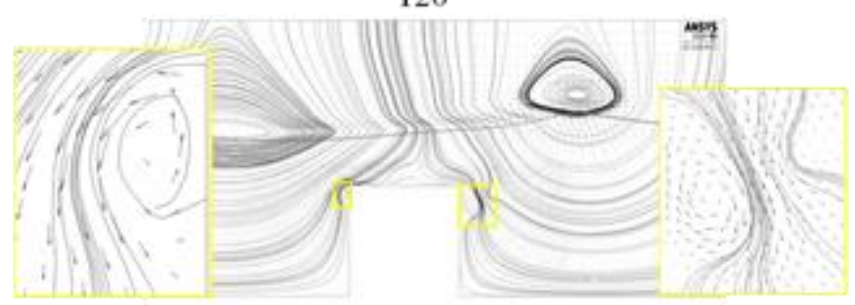

$180^{\circ}$

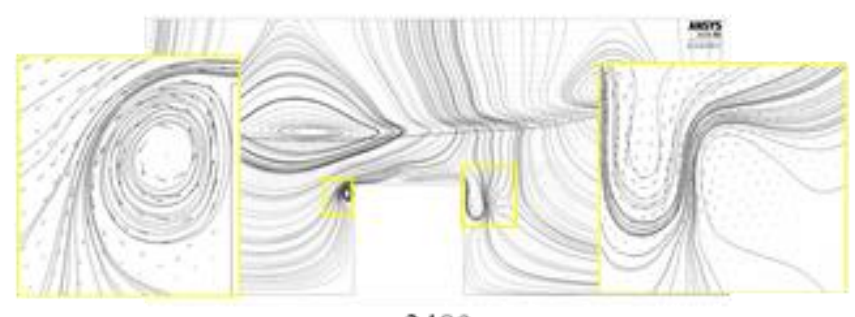

$240^{\circ}$
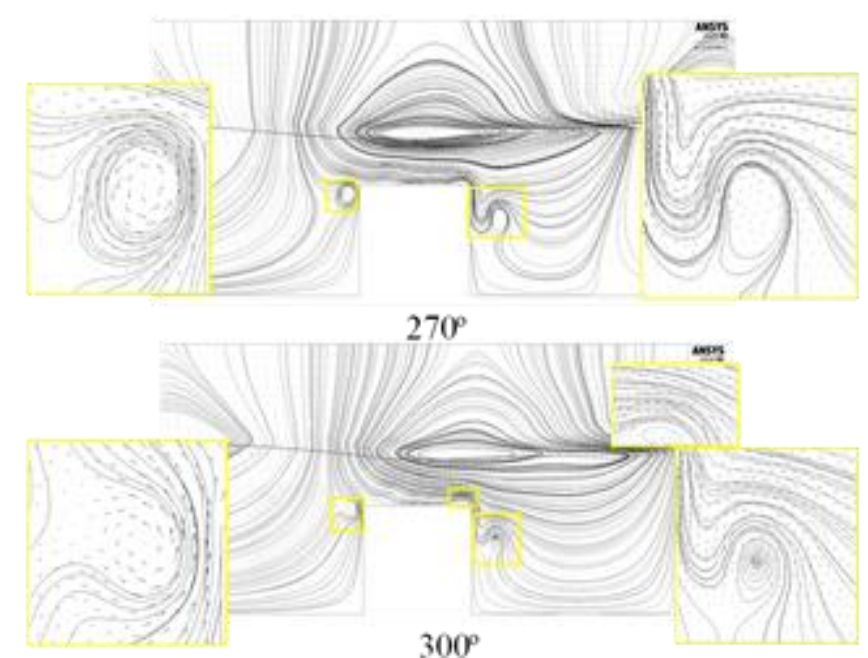

Fig. 14. Streamlines at 6 significant phases, case 9

\subsection{Wave Drag and Moment}

The wave forces affecting the obstacle are calculated numerically for the nine cases listed in Table

3. The pressure drag is defined as $D=F_{1}-F_{2}$, where $F_{a}=\int_{0}^{L} p_{a} d l$. The variables are explained in Figure 15. The maximum drag values are provided in Figure 16 and 17, in the anti-wave (negative) 
and wave propagation (positive) directions, respectively. The maximum value is calculated over one wave period. It should be noted that absolute values are plotted.

The anti-wave drag values are considerably higher than those in the wave direction. The phenomenon of higher anti-wave drag is well known and was fully explained in pages 142,143 and 144 of [1]. The maximum values of resultant turning moment due to pressure are shown in Figure 18. The rotation is assumed positive in clockwise direction. It is predicated that the maximum resultant moment is always in the anti-clock direction. This is consistent with the calculated drag results, where failure was anticipated to be in the anti-wave direction.

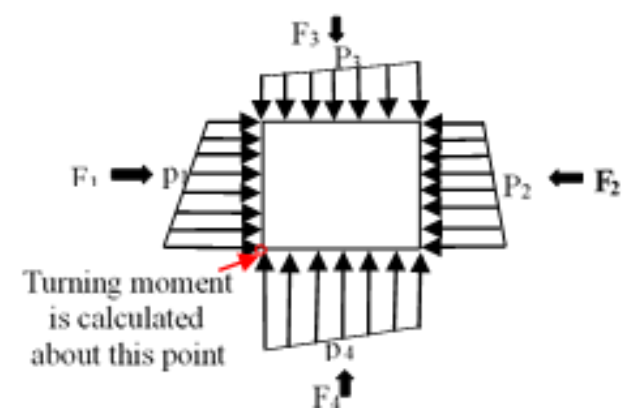

Fig. 15. Pressure forces acting on the square obstacle
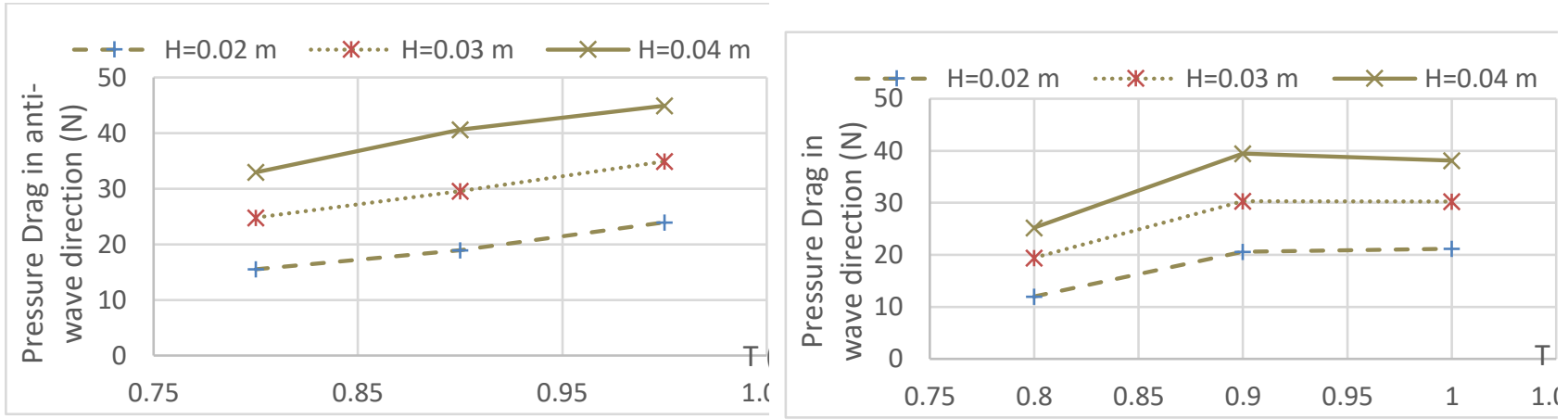

Fig. 16. Pressure Drag on the obstacle (anti-wave) Fig. 17. Pressure Drag on the obstacle vs. wave vs. wave period period

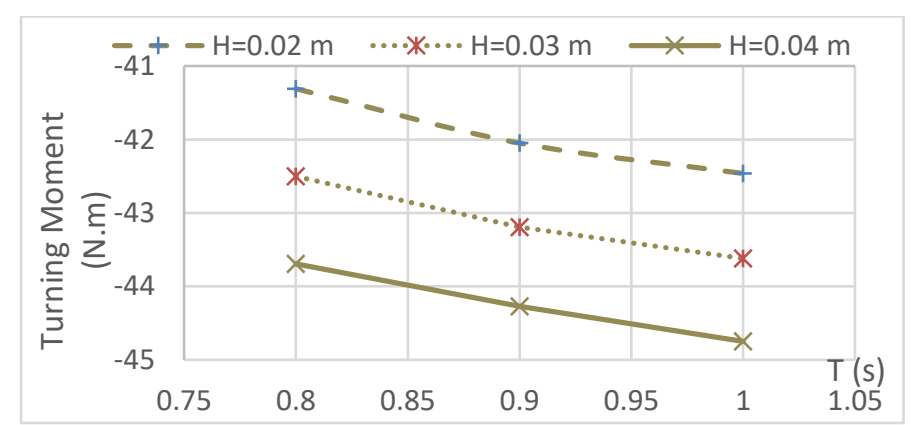

Fig. 18. Turning Moment acting on the square obstacle. (-ve means anticlockwise) 


\section{Conclusions}

The problem of wave propagation of water waves over a large submerged square obstacle is simulated. Various challenges regarding turbulence, flow field resolution and initial and boundary conditions are well addressed and described. The results are extensively validated using velocity field measurements. Temporal and spatial accuracy is illustrated using vertical and horizontal velocity profiles. The complex wave induced vortical structure is captured. Specifically, four distinct separation vortices are fully visualized and explained. Finally, the numerical results are used to compute the wave drag and moment for a brief parametric study. The proportional relation between wave period and drag forces is illustrated. The failure in anti-wave direction is predicted, as consistent with current literature. The authors plan to extend the numerical model to other shapes and types of obstacles, pursuing an optimum design.

\section{References}

[1] Goda, Yoshimi. Random seas and design of maritime structures. Vol. 33. World Scientific Publishing Company, 2010. https://doi.org/10.1142/7425

[2] Johnson, Hakeem K., Theophanis V. Karambas, Ioannis Avgeris, Barbara Zanuttigh, Daniel Gonzalez-Marco, and I. Caceres. "Modelling of waves and currents around submerged breakwaters." Coastal Engineering 52, no. 10-11 (2005): 949-969. https://doi.org/10.1016/i.coastaleng.2005.09.011

[3] Zuan, A. M. S., M. K. Z. Anuar, S. Syahrullail, M. N. Musa, and E. A. Rahim. "A study of float wave energy converter (FWEC) model." Journal of Advanced Research in Applied Sciences and Engineering Technology 1, no. 1 (2015): 4049.

[4] Chang, Kuang-An, Tian-Jian Hsu, and Philip L-F. Liu. "Vortex generation and evolution in water waves propagating over a submerged rectangular obstacle: Part I. Solitary waves." Coastal Engineering 44, no. 1 (2001): 13-36. https://doi.org/10.1016/S0378-3839(01)00019-9

[5] Kasem, Tamer Heshmat Mohamed Aly. "Development of a Generic Numerical Wave Tank Coupling Solid, Liquid and Air." PhD diss., Yokohama National University, 2010.

[6] Cooker, M. J., D. H. Peregrine, C. Vidal, and J. W. Dold. "The interaction between a solitary wave and a submerged semicircular cylinder." Journal of Fluid Mechanics 215 (1990): 1-22. https://doi.org/10.1017/S002211209000252X

[7] Fluent, A. N. S. Y. S. "ANSYS Fluent User's Guide r15." Chapter 6 (2013): 223-247.

[8] Fluent, A. N. S. Y. S. "ANSYS fluent theory guide 15.0." ANSYS, Canonsburg, PA 33 (2013).

[9] Ahmed, Mushtaq, Zafarullah Nizamani, Akihiko Nakayama, and Montasir Osman. "Some Recent Fluid-Structure Interaction Approaches for the Wave Current Behaviour With Offshore Structures." CFD Letters 12, no. 9 (2020): 15-26. https://doi.org/10.37934/cfdl.12.9.1526

[10] A. Seeni, P. Rajendran, and H. Mamat. "A CFD Mesh Independent Solution Technique for Low Reynolds Number Propeller." CFD Letters 11, no. 10 (2019): 15-30.

[11] Yuan, Dekui, and Jianhua Tao. "Wave forces on submerged, alternately submerged, and emerged semicircular breakwaters." Coastal Engineering 48, no. 2 (2003): 75-93. https://doi.org/10.1016/S0378-3839(02)00169-2

[12] Sun, Jiawen, Zhe Ma, Dongxu Wang, Sheng Dong, and Ting Zhou. "Numerical study of the run-up of a solitary wave after propagation over a saw-tooth-shaped submerged breakwater." International Journal of Naval Architecture and Ocean Engineering 12 (2020): 283-296. https://doi.org/10.1016/i.ijnaoe.2019.11.002

[13] Ji, Qiaoling, Yu Wang, and Guowei Zhang. "Numerical study of solitary wave interaction with a submerged semicircular cylinder." Mathematical Problems in Engineering 2019 (2019). https://doi.org/10.1155/2019/3589052

[14] Fathi, Amin, and Mohammad Javad Ketabdari. "Modeling of emerged semi-circular breakwater performance against solitary waves using SPH method." Journal of the Brazilian Society of Mechanical Sciences and Engineering 40, no. 6 (2018): 1-14. https://doi.org/10.1007/s40430-018-1179-4

[15] T. Kasem, A. Schlenkhoff, and S. Peterseim. "Measurement of Wave Induced Flow Behind Submerged Obstacles Using A Custom PIV System." In 5th International Conference on The Application of Physical Modelling to Port and Coastal Protection, Varna, Bulgaria, 2014.

[16] Menter, Florian R. "Two-equation eddy-viscosity turbulence models for engineering applications." AIAA journal 32 , no. 8 (1994): 1598-1605. https://doi.org/10.2514/3.12149

[17] White, F. M., and G. H. Christoph. A simple new analysis of compressible turbulent two-dimensional skin friction under arbitrary conditions. Rhode Island Univ Kingston Dept of Mechanical Engineering and Applied Mechanics, 
1971.

[18] Elhanafi, Ahmed, Alan Fleming, Zhi Leong, and Gregor Macfarlane. "Effect of RANS-based turbulence models on nonlinear wave generation in a two-phase numerical wave tank." Progress in Computational Fluid Dynamics, an International Journal 17, no. 3 (2017): 141-158. https://doi.org/10.1504/PCFD.2017.084350

[19] Dean, Robert G., and Robert A. Dalrymple. Water wave mechanics for engineers and scientists. Vol. 2. World Scientific Publishing Company, 1991. https://doi.org/10.1142/1232

[20] Sveen, Johan Kristian. "An introduction to MatPIV v. 1.6. 1." Preprint series. Mechanics and Applied Mathematics http://urn. nb. no/URN: NBN: no-23418 (2004). 